\title{
Qualidade visual após retratamento de LASIK personalizado versus convencional
}

\author{
Visual quality after custom versus standard LASIK retreatment
}

\author{
Andréia Peltier Urbano ${ }^{1}$ \\ Walton Nosé ${ }^{2}$
}

Trabalho realizado no Departamento de Oftalmologia da Universidade Federal de São Paulo - UNIFESP - São Paulo (SP) - Brasil.

Doutora em Medicina pelo Curso de Pós-Graduação em Oftalmologia e Ciências Visuais da Universidade Federal de São Paulo - UNIFESP - São Paulo (SP) - Brasil.

${ }^{2}$ Livre Docente, Professor do Departamento de Oftalmologia da UNIFESP - São Paulo (SP) - Brasil; Professor Titular do Departamento de Oftalmologia da Universidade Metropolitana de Santos - UNIMES - Santos (SP) - Brasil

Endereço para Correspondência: Andréia Peltier Urbano. Av. Cardeal da Silva 2070/201B - Salvador (BA) CEP 40231-250

E-mail: andreiaurbano@terra.com.br

Recebido para publicação em 21.08.2007

Ultima versão recebida em 26.06.2008

Aprovação em 13.08.2008

Nota Editorial: Depois de concluída a análise do artigo sob sigilo editorial e com a anuência da Dra. Belquiz Rodrigues A. Nassaralla sobre a divulgação de seu nome como revisora, agradecemos sua participação neste processo.

\section{RESUMO}

Objetivo: Avaliar a qualidade visual após retratamento personalizado e retratamento convencional em olhos submetidos a LASIK primário convencional. Métodos: Foi realizado estudo prospectivo de 74 olhos submetidos ao retratamento de LASIK para correção de miopia e astigmatismo. Cada paciente foi submetido ao retratamento de LASIK com ablação personalizada (Zyoptix, Bausch \& Lomb) em um olho e ablação convencional (Planoscan, Bausch \& Lomb) no olho contralateral. Foram comparados os resultados do teste de ofuscamento e da sensibilidade ao contraste durante o acompanhamento de seis meses. Resultados: Os olhos do grupo Zyoptix apresentaram melhores resultados no teste de ofuscamento e na sensibilidade ao contraste do que o olho contralateral, quando se compararam os resultados. Conclusões: O retratamento personalizado resultou em melhor qualidade visual pós-operatória do que o retratamento convencional de LASIK primário convencional.

Descritores: Qualidade; Visão; Ceratomileuse assistida por excimer laser in situ; Retratamento; Lasers; Miopia/cirurgia; Erros de refração/cirurgia

\section{INTRODUÇ̃̃̃O}

Distúrbios de visão noturna com sintomas de ofuscamento e halos em torno da luz são comuns após a cirurgia refrativa com excimer laser, sendo descrita uma incidência que varia, na literatura, de 3 a $60 \%{ }^{(1-2)}$. Diversos estudos evidenciam alteração da qualidade visual com decréscimo da sensibilidade ao contraste no pós-operatório ${ }^{(3-4)}$.

Em fase do avanço no campo da análise de frente de onda, já está bem estabelecido que as principais causas associadas aos distúrbios de visão noturna estão relacionadas ao aumento das aberrações oculares monocromáticas e ao fenômeno da dispersão da luz através da córnea operada ${ }^{(5-6)}$.

Um dos focos atuais no estudo da cirurgia refrativa tem sido a de demonstrar a correlação entre o aumento das aberrações e a alteração de exames da qualidade visual. Já foi bem evidenciado que existe correlação entre a magnitude das aberrações oculares e a acuidade visual de alto e baixo contraste, entre a acuidade visual com teste de ofuscamento e sensibilidade ao contraste ${ }^{(7-8)}$.

Como as atividades visuais noturnas são predominantemente realizadas sob condições de contraste reduzido e em baixas freqüências espaciais, os testes visuais mais adequados para a avaliação dos distúrbios da visão noturna utilizam condições escotópicas ou mesópicas, com baixo contraste. Os testes de alto contraste, como o teste da acuidade visual com a tabela de Snellen, geralmente não são sensíveis para evidenciar as alterações da qualidade visual $^{(8-10)}$. 
Embora a cirurgia refrativa convencional ofereça bons resultados de acuidade visual, acredita-se que a nova geração de tratamentos personalizados irá preservar e até melhorar a qualidade visual que historicamente tem sido afetada com os tratamentos convencionais, particularmente sob condições mesópicas e escotópicas ${ }^{(11-12)}$. Ao proporcionar a correção das aberrações de baixa ordem, sem ou reduzir as aberrações de alta ordem, a cirurgia personalizada produz uma superfície ocular mais homogênea, com melhor qualidade visual.

Como na literatura não há estudos comparativos da qualidade visual após o retratamento com as técnicas personalizada e convencional, optamos por avaliar qual tratamento proporciona melhor qualidade visual após o retratamento de LASIK primário convencional.

\section{OBJETIVO}

Comparar os resultados do teste de ofuscamento e de sensibilidade ao contraste de olhos submetidos ao retratamento personalizado e convencional de LASIK.

\section{MÉTODOS}

Com base em um estudo prospectivo e randomizado, 74 olhos foram submetidos a retratamento de LASIK para correção de miopia e astigmatismo.

O grupo de estudo constituiu-se de pacientes que tiveram um dos olhos submetido a retratamento de LASIK pela técnica de LASIK com ablação personalizada Zyoptix (Bausch \& Lomb, Munique, Baviera). O grupo-controle foi composto pelo olho contralateral submetido a retratamento pela técnica de LASIK com ablação convencional Planoscan (Bausch \& Lomb, Munique, Baviera). A escolha do olho a ser submetido ao retratamento com a técnica personalizada ou convencional foi aleatória.

Os pacientes realizaram cirurgia de LASIK primária com a técnica convencional, no período de novembro de 1995 a fevereiro de 2003, por um único cirurgião, na Eye Clinic Day Hospital (São Paulo, Brasil). Os retratamentos de LASIK foram realizados, no período de agosto de 2002 a outubro de 2003, com levantamento do disco da cirurgia anterior, por um único cirurgião. Após a cirurgia de retratamento de LASIK, os pacientes foram acompanhados por um período de seis meses.

Todos os pacientes assinaram consentimento informado e o estudo foi aprovado pelo Comitê de Ética da Universidade Federal de São Paulo.

No pré-operatório, os pacientes realizaram um exame completo incluindo teste de ofuscamento ("glare") com o aparelho Brightness Acuity Test (BAT) (Marco Ophthalmic Inc., Jacksonville, Florida) e tabela ETDRS com acuidade visual de baixo contraste e teste de sensibilidade ao contraste com a Tabela CSV 1000E (Vector Vision, Daytona, Ohio).

No período pós-operatório, os pacientes usaram colírios de tobramicina a $0,3 \%$, de cetrolaco de trometamina a $0,5 \%$, de acetato de prednisolona a $0,12 \%$ e de hipromelose a $0,5 \%$, sendo examinados no primeiro dia e aos 30, 90 e 180 dias após a cirurgia. Toda a avaliação pré e pós-operatória foi realizada por um mesmo examinador.

\section{Método estatístico}

A análise estatística foi realizada com o Programa Statistical Package for the Social Sciences SPSS (SPSS Inc., Chicago, Illinois) na versão 10.0 para Windows.

Os resultados da acuidade visual de baixo contraste obtidos com o teste de ofuscamento sem luz, com luz fraca, luz média e luz forte foram descritos como média e como desviopadrão em unidades decimais.

Para verificação da similaridade dos dados pré-operatórios entre o grupo Zyoptix (grupo de estudo) e o grupo Planoscan (grupo-controle), foram procedidos os testes estatísticos de Mann-Whitney para as seguintes variáveis: do equivalente esférico (EE) da refração clínica dinâmica; das aberrações oculares (baixa e alta ordens e totais), mensuradas com o aberrômetro Zywave (Bausch \& Lomb, Munique, Baviera), considerando-se o diâmetro da pupila de $6,0 \mathrm{~mm}$; da acuidade visual de baixo contraste, medida com o teste de ofuscamento sem luz, com luz fraca, média e forte, bem como da sensibilidade ao contraste para frequiências espaciais de 3, 6, 12 e 18 ciclos/ graus.

O teste de Wilcoxon foi utilizado para avaliar a modificação da acuidade visual de baixo contraste com o teste de ofuscamento entre os períodos pré-operatório e pós-operatório de um mês, três meses e seis meses, separadamente, para os grupos Zyoptix e Planoscan. O teste de Mann-Whitney foi realizado para comparar as médias dos resultados entre os grupos Zyoptix e Planoscan em cada período do estudo.

Os resultados do teste de sensibilidade ao contraste apresentados em forma de gráfico da sensibilidade ao contraste (descrita em unidades logarítmicas) versus a freqüência espacial (descrito em ciclos por grau). O valor da sensibilidade ao contraste foi expresso na notação dos valores da sensibilidade ao contraste normalizados e desenvolvidos por Boxer Wachler et al. ${ }^{(13)}$. Para a normalização, o valor da sensibilidade ao contraste foi dividido pelo valor da média populacional de indivíduos normais da mesma faixa etária, também descritos por Boxer Wachler et al. ${ }^{(13)}$. Os valores da sensibilidade ao contraste normalizados para cada freqüência espacial foram descritos como média e desvio-padrão. O teste de Wilcoxon foi usado para avaliar as modificações das médias da sensibilidade ao contraste no pré-operatório e pós-operatório, separadamente, para os grupos Zyoptix e Planoscan. O teste de Mann-Whitney foi utilizado para comparar os valores da sensibilidade ao contraste entre os grupos Zyoptix e Planoscan para cada período estudado. 


\section{RESULTADOS}

A análise dos dados do período pré-operatório do retratamento de LASIK evidenciou que os olhos dos grupos Zyoptix e Planoscan foram similares quanto aos valores do EE da refração clínica; das aberrações oculares de baixa e alta ordens e aberrações totais; da acuidade visual de baixo contraste medida com o teste de ofuscamento sem luz, com luz fraca, média e forte; bem como da sensibilidade ao contraste para freqüências espaciais de 3, 6, 12 e 18 ciclos/graus ( $>0,05)$, conforme pode ser verificado na tabela 1 .

\section{Teste de ofuscamento com o BAT}

Os resultados dos testes de ofuscamento com o BAT realizados no período pré-operatório e no acompanhamento de um mês, três meses e seis meses, nos grupos Zyoptix e Planoscan, estão apresentados, respectivamente, nas figuras 1 e 2 .

A análise de figura 1 permite observar que os valores da acuidade visual de baixo contraste no grupo Zyoptix foram superiores no período pós-operatório para todas as condições de ofuscamento testados. A maior diferença entre a acuidade visual do pré-operatório, em relação ao pós-operatório, ocorreu nas condições de ofuscamento com luz média e forte.

A análise da figura 2 permite observar que os valores da acuidade visual de baixo contraste, no grupo Planoscan, foram muito próximos no período pós-operatório em relação ao pré-operatório para todas as condições de ofuscamento testadas. A menor diferença entre a acuidade visual do pré-operatório em relação ao pós-operatório, se verificou na condição de ofuscamento com luz forte. No período de um mês, a média da acuidade visual de baixo contraste foi inferior à média do pré-operatório. Nos períodos de três e seis meses, os valores foram equivalentes aos do pré-operatório.

\section{Teste de sensibilidade ao contraste}

Os resultados dos testes de sensibilidade ao contraste avaliados no período pré-operatório e no acompanhamento de um, três e seis meses nos grupos Zyoptix e Planoscan, separadamente, estão apresentados nas figuras 3 e 4.

A análise da figura 3 permite observar que no grupo Zyoptix houve melhora dos valores da sensibilidade ao contraste para todas as frequiências espaciais em todos os períodos pósoperatórios avaliados. Pode-se, também, observar que os valores da sensibilidade ao contraste foram semelhantes às frequiências espaciais de 3, 6 e 12 ciclos/graus (cpg), em todos os períodos pós-operatórios. A freqüência espacial de $18 \mathrm{cpg}$ foi a que apresentou o maior aumento em relação ao valor préoperatório.

A análise da figura 4 permite observar que, no grupo Planoscan, os valores da sensibilidade ao contraste, no período pré-operatório e pós-operatório, foram próximos. A média da sensibilidade ao contraste para as freqüências espaciais de $12 \mathrm{e}$ 18 ciclos/graus, no período de um mês, foi igual à média do pré-operatório, com melhora nos períodos de três e seis meses.

\section{DISCUSSÃO}

Para se comparar a qualidade visual de olhos submetidos à cirurgia refrativa é importante escolher exames adequados que possam evidenciar esta função, especialmente quando o estudo objetiva evidenciar se um tipo de foto-ablação proporciona melhor qualidade visual.

Embora o clássico exame da acuidade visual com a tabela de Snellen seja utilizado para avaliação do ganho ou perda de quantidade visual na grande parte dos estudos, como este mensura apenas a variável tamanho do objeto e utiliza contraste

Tabela 1. Análise comparativa do período pré-operatório do retratamento de LASIK entre os grupos Zyoptix e Planoscan

\begin{tabular}{|c|c|c|c|}
\hline Número de olhos & $\begin{array}{c}\text { Grupo Zyoptix } \\
37\end{array}$ & $\begin{array}{c}\text { Grupo Planoscan } \\
37\end{array}$ & $\mathbf{p}$ \\
\hline EE da refração clínica (D) & $-1,34 \pm 0,81$ & $-1,33 \pm 0,74$ & 0,91 \\
\hline Baixa ordem & $3,20 \pm 1,45$ & $3,13 \pm 1,12$ & 0,87 \\
\hline Alta ordem & $0,77 \pm 0,33$ & $0,77 \pm 0,31$ & 0,87 \\
\hline Aberrações totais & $3,31 \pm 1,44$ & $3,24 \pm 1,12$ & 0,83 \\
\hline Luz fraca & $0,74 \pm 0,22$ & $0,75 \pm 0,19$ & 0,60 \\
\hline Luz média & $0,70 \pm 0,24$ & $0,75 \pm 0,19$ & 0,36 \\
\hline Luz forte & $0,65 \pm 0,26$ & $0,69 \pm 0,18$ & 0,44 \\
\hline \multicolumn{4}{|c|}{ Teste de sensibilidade ao contraste (log) } \\
\hline Freqüência espacial 3 cpg & $0,93 \pm 0,11$ & $0,97 \pm 0,12$ & 0,25 \\
\hline Freqüência espacial 6 cpg & $0,95 \pm 0,09$ & $0,94 \pm 0,12$ & 0,93 \\
\hline
\end{tabular}




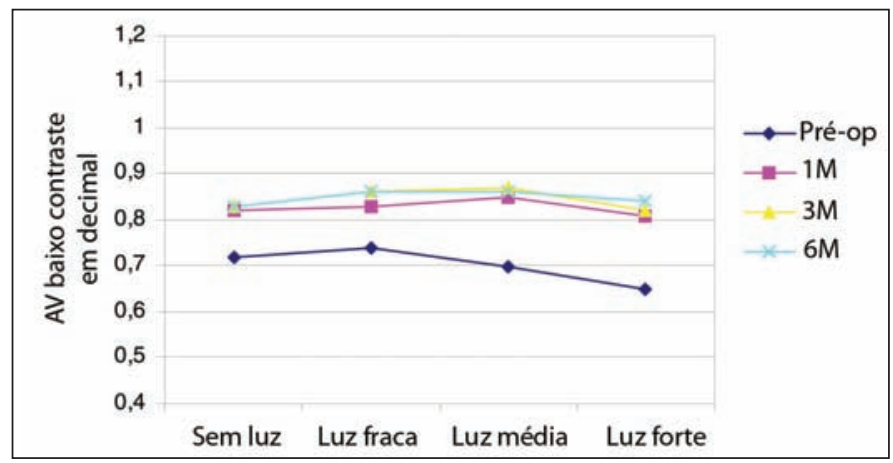

Figura 1 - Acompanhamento do teste de ofuscamento (BAT) no grupo Zyoptix

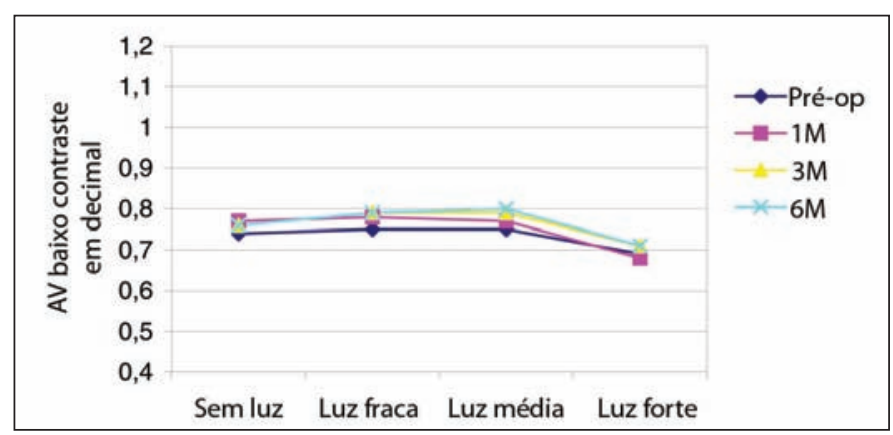

Figura 2 - Acompanhamento do teste de ofuscamento (BAT) no grupo Planoscan

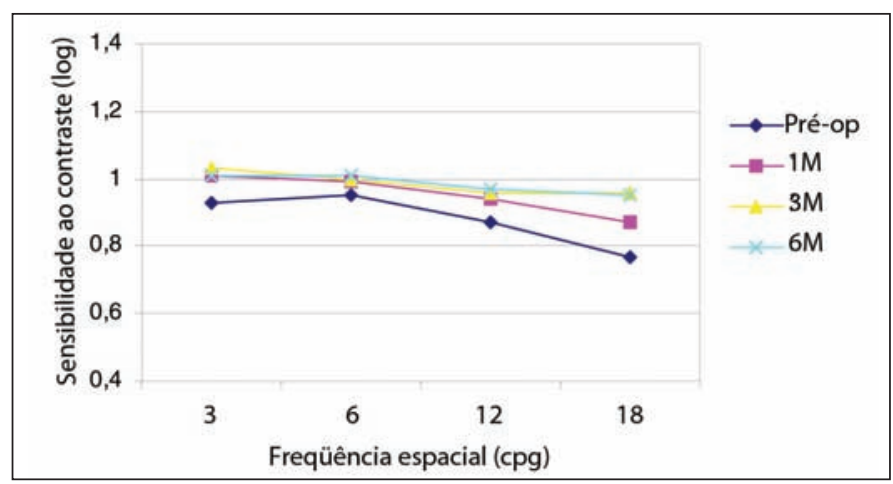

Figura 3 - Acompanhamento do teste de sensibilidade ao contraste no grupo Zyoptix

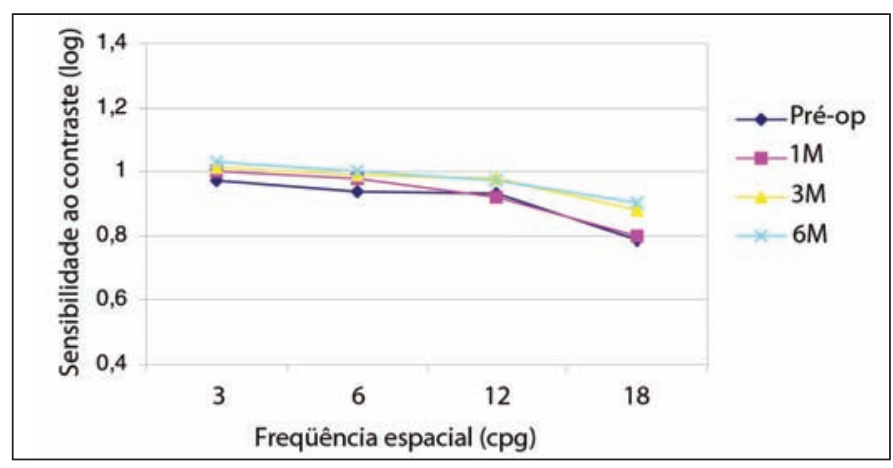

Figura 4 - Acompanhamento do teste de sensibilidade ao contraste no grupo Planoscan alto, próximo a $100 \%$, não é um exame indicado para se comparar a qualidade visual ${ }^{(8-10)}$. Também a simples comparação do valor das aberrações oculares, medido em unidade de RMS ("root mean squared error"), também não é um bom indicador para representar a qualidade da imagem formada por estas aberrações, pois este é apenas um índice quantitativo e não qualitativo ${ }^{(6-7)}$.

Os testes de ofuscamento e os testes de sensibilidade visual estão entre os mais indicados para avaliação qualitativa, pois além de avaliarem a variável tamanho do objeto, também avaliam o contraste do objeto ${ }^{(14)}$. O teste de ofuscamento com o aparelho BAT (Marco Ophthalmic Inc., Jacksonville, Florida) é utilizado para medir o ofuscamento sob condições que mimetizam o ofuscamento diurno e avalia o comportamento da acuidade visual funcional em três condições de luz ofuscante $^{(8)}$. A maior parte dos estudos que realizaram o teste de ofuscamento utilizou tabelas de acuidade visual de Snellen de alto e baixo contraste ou tabelas de sensibilidade ao contraste ${ }^{(14)}$. Como a acuidade visual de baixo contraste é um parâmetro mais sensível do que a acuidade visual de alto contraste para identificação de mudanças discretas na qualidade visual $^{(15)}$, optou-se por utilizar a tabela da acuidade visual de baixo contraste do ETDRS, associada ao teste de ofuscamento, para mensuração da qualidade visual no retratamento de LASIK. A avaliação da qualidade visual através de testes de sensibilidade ao contraste com grades senoidais é atualmente um dos métodos mais sensíveis e utilizados nos estudos científicos $^{(14-18)}$. O teste com o CSV-1000 (Vector Vision, Dayton, Ohio) vem sendo utilizado por muitos autores e apresenta valores com confiabilidade e repetibilidade para a cirurgia refrativa ${ }^{(14)}$.

$\mathrm{Na}$ análise dos resultados do teste de ofuscamento, podese observar que, no grupo Zyoptix houve uma melhora estatisticamente significativa da acuidade visual de baixo contraste em todos os períodos pós-operatórios. A análise da figura 1 revela que, no período pré-operatório, a média da acuidade visual de baixo contraste apresentava uma queda, principalmente nas condições de luminância média e forte. Isto sugere que os pacientes apresentavam perda da qualidade visual sob condições de maior luminância. Desde o primeiro mês do pós-operatório, houve uma melhora significativa da acuidade visual de baixo contraste para todas as luminâncias avaliadas e não mais se constatou queda da acuidade visual nas condições de luminância média e forte. Com base nesta análise, pode-se inferir que, após a cirurgia de retratamento personalizada Zyoptix, a qualidade visual é mantida sob todas as condições de luminosidade testadas.

Quanto aos resultados do teste de ofuscamento no grupo Planoscan, nota-se que os valores da acuidade visual de baixo contraste foram discretamente superiores no período pósoperatório em relação ao período pré-operatório, sem diferença estatística. A avaliação da figura 2 revela que a tendência da queda da acuidade visual encontrada no período préoperatório, para condições de luminância forte, também foi mantida no período pós-operatório. Com isto, é possível infe- 
rir que essa perda da qualidade visual sob condições de luminância forte no período pré-operatório não foi recuperada após o retratamento convencional Planoscan.

Quando se analisam os resultados da sensibilidade ao contraste neste estudo, observou-se que no grupo Zyoptix houve uma melhora estatística da sensibilidade ao contraste para todas as frequiências espaciais avaliadas para todos os períodos pós-operatórios comparados em relação aos valores pré-operatórios. Na figura 3, pode-se verificar que no período pré-operatório mais queda da curva da sensibilidade ao contraste, ocorreu para as frequiências espaciais mais altas de 12 e 18 ciclos/ grau. No período de um mês, apesar da melhora significativa dos valores da sensibilidade ao contraste para todas as frequiências, ainda pode ser observada uma discreta queda na curva da sensibilidade para as frequiências de 12 e 18 ciclos/grau. A partir do terceiro mês, não houve mais a queda da curva da sensibilidade ao contraste e as médias da sensibilidade ao contraste para todas as frequiências espaciais ficaram mais próximas. Isto é evidenciado pela curva da sensibilidade, que tende a parecer mais uma reta, com todos os valores mais próximos a um. Com esta análise, fica claro que, após a cirurgia de retratamento personalizado Zyoptix, os olhos apresentaram um ganho de qualidade visual, principalmente para as frequiências espaciais mais altas, que obtiveram sensibilidades ao contraste comparáveis com as sensibilidades para as frequiências espaciais mais baixas. A melhora da sensibilidade ao contraste, principalmente para freqüências espaciais mais altas, pode ser apoiada pelas constatações de Montes-Micó et al. ${ }^{(19)}$, que enfatizaram que o defocus e as aberrações ópticas primariamente afetam as freqüências espaciais altas.

Quanto aos resultados do teste de sensibilidade ao contraste no grupo Planoscan, observou-se uma melhora estatística dos valores em relação ao pré-operatório para a frequiência de 6 ciclos/grau; a partir do primeiro mês do pós-operatório, para as frequiências de 3 e 18 ciclos/grau; apenas a partir do terceiro mês do pós-operatório, bem como para freqüência de 12 ciclos/grau, os valores pós-operatórios não apresentaram diferença estatística em relação ao pré-operatório. A análise da figura 4 permite observar que a curva da sensibilidade ao contraste no pós-operatório, no período de um mês, era muito parecida com a curva pré-operatória. Apesar do aumento dos valores da sensibilidade ao contraste nos pós-operatórios de três e seis meses, ainda pode-se notar uma discreta queda da sensibilidade ao contraste para a freqüência espacial de 18 ciclos/grau. Em face desses dados, pode-se inferir que, após o retratamento convencional Planoscan, ainda há uma discreta tendência de alteração da sensibilidade ao contraste para a frequiência espacial mais alta.

É interessante observar que a tendência das curvas da sensibilidade ao contraste foi semelhante à tendência das curvas do teste de ofuscamento, separadamente para os grupos Zyoptix e Planoscan. Essa similaridade da qualidade visual obtida com dois tipos de testes diferentes permite uma maior validação dos resultados encontrados.
É por demais conhecido que, após a cirurgia de LASIK primária convencional, pode haver uma diminuição temporária da sensibilidade ao contraste, sob condições fotópicas, mesópicas e escotópicas ${ }^{(8,20-21)}$. Alguns estudos demonstram que a recuperação da sensibilidade ao contraste para níveis similares aos do pré-operatório, pode ocorrer precocemente no primeiro mês do pós-operatório ${ }^{(21)}$, enquanto outros autores relataram a recuperação dentro do período de três e doze meses após a cirurgia ${ }^{(22)}$. Alguns autores reportaram redução da sensibilidade ao contraste após o LASIK primário convencional principalmente para frequiências mais baixas de 3 e 6 ciclos/grau ${ }^{(23)}$, enquanto outros observaram esta redução para frequiências mais altas de 12 e 18 ciclos/grau ${ }^{(8,21)}$.

$\mathrm{Na}$ maior parte dos estudos que avaliaram a sensibilidade ao contraste em olhos submetidos à cirurgia de LASIK primária personalizada, houve uma tendência de melhora significativa da sensibilidade ao contraste no pós-operatório. Alguns estudos verificaram melhora para uma ou mais frequiências espaciais testadas ${ }^{(24-28)}$, enquanto outros observaram melhora para todas as frequiências espaciais ${ }^{(17,26)}$. Essas melhoras da sensibilidade ao contraste foram observadas para testes realizados sob condições fotópicas ${ }^{(24-26)}$, mesópicas ${ }^{(24,26)}$ e escotópicas ${ }^{(17)}$.

Quando comparados os resultados dos testes de sensibilidade ao contraste em olhos submetidos à cirurgia de LASIK primária com as técnicas personalizada e convencional, podese observar uma tendência de melhores resultados com a cirurgia personalizada. No estudo de Kaiserman et al. ${ }^{(29)}$, os autores verificaram que, após um mês da cirurgia de LASIK primária personalizada com a plataforma Allegretto (WaveLight Laser Technologie AG, Alemanha), houve um aumento médio da sensibilidade ao contraste de $34,3 \pm 4,3 \%$, enquanto após a cirurgia convencional o aumento foi de apenas $1,5 \pm 3,2 \%$. A diferença de melhora da sensibilidade ao contraste foi estatisticamente significativa para todas as frequiências espaciais, exceto para a freqüência de 6 ciclos/grau.

Merece ser registrado um aspecto interessante constatado neste estudo: os valores da sensibilidade ao contraste no retratamento convencional Planoscan não apresentaram redução após a cirurgia conforme se esperava após a cirurgia de LASIK primária convencional. Pelo contrário, houve uma melhora estatística da sensibilidade ao contraste para as freqüências de 3, 6 e 18 ciclos/grau. Desse modo, entende-se que a cirurgia de retratamento de LASIK convencional pode proporcionar uma melhor qualidade visual do que a cirurgia de LASIK primária. Esse pensamento pode ser apoiado pelos resultados encontrados no estudo de Rani et al. ${ }^{(30)}$. Estes autores observaram que, após o retratamento de LASIK convencional para correção de equivalente esférico de $-2,85 \pm$ 2,17 D com a plataforma Planoscan, não houve mudança significativa da sensibilidade ao contraste, diferentemente do LASIK primário convencional, que apresentou uma diminuição estatística para os valores da sensibilidade ao contraste ${ }^{(30)}$. Deste modo pode-se notar que o retratamento de LASIK primário tende a ser superior à cirurgia de LASIK primária no que se refere à qualidade visual pós-operatória. 


\section{CONCLUSÕES}

O retratamento de LASIK primário convencional com ablação personalizada resultou em melhor qualidade visual pós-operatória do que o retratamento com ablação convencional, apresentando uma melhora estatisticamente significativa dos valores da acuidade visual de baixo contraste com o teste de ofuscamento e da sensibilidade ao contraste durante o acompanhamento de seis meses.

\section{AGRADECIMENTOS}

Agradecimento à Direção, colegas e funcionários da Eye Clinic pelo grande apoio e importante colaboração neste estudo.

\section{ABSTRACT}

Purpose: To evaluate visual quality after wavefront-guided LASIK versus standard LASIK in retreatment of primary LASIK for myopia and myopic astigmatism. Methods: A prospective study was performed with paired eye control of 74 eyes with LASIK retreatment. Each patient underwent retreatment using custom ablation (Zyoptix, Bausch \& Lomb) in 1 eye and standard ablation LASIK (PlanoScan, Bausch \& Lomb) in the contralateral eye. A complete ophthalmologic examination was performed, including evaluation of glare test and contrast sensitivity test, with a follow-up of 6 months. Results: Zyoptix eyes showed better results of glare test and contrast sensitivity test than the contralateral eyes. Conclusions: Wavefront-guided LASIK produces better visual quality than standard LASIK in the retreatment of refractive errors after primary LASIK.

Keywords: Quality; Vision; Keratomileusis, laser in situ; Retreatment; Lasers; Myopia/surgery; Refractive errors/surgery

\section{REFERÊNCIAS}

1. Hamberg-Nyström H, Tengroth B, Fagerholm P, Epstein D, van der Kwast EM. Patient satisfaction following photorefractive keratectomy for myopia. J Refract Surg. 1995;11(Suppl 3):S335-6.

2. Halliday BL. Refractive and visual results and patient satisfaction after excimer laser photorefractive keratectomy for myopia. Br J Ophthalmol. 1995;79(10): 881-7. Comment in: Br J Ophthalmol. 1996;80(4):380.

3. Schlote T, Kriegerowski M, Bende T, Derse M, Thiel HJ, Jean B. Mesopic vision in myopia corrected by photorefractive keratectomy, soft contact lenses, and spectacles. J Cataract Refract Surg. 1997;23(5):718-25.

4. Niesen U, Businger U, Hartmann P, Senn P, Schipper I. Glare sensitivity and visual acuity after excimer laser photorefractive keratectomy for myopia. Br J Ophthalmol. 1997;81(2):136-40.

5. Moreno-Barriuso E, Lloves JM, Marcos S, Navarro R, Llorente L, Barbero S. Ocular aberrations before and after myopic corneal refractive surgery: LASIKinduced changes measured with laser ray tracing. Invest Ophthalmol Vis Sci. 2001;42(6):1396-403.

6. Marcos S. Aberrations and visual performance following standard laser vision correction. J Refract Surg. 2001;17(5):S596-601.
7. Holladay JT, Dudeja DR, Chang J. Functional vision and corneal changes after laser in situ keratomileusis determined by contrast sensitivity, glare testing, and corneal topography. J Cataract Refract Surg. 1999;25(5):663-9.

8. Mutyala S, McDonald MB, Scheinblum KA, Ostrick MD, Brint SF, Thompson $\mathrm{H}$. Contrast sensitivity evaluation after laser in situ keratomileusis. Ophthalmology. 2000;107(10):1864-7.

9. Pérez-Santonja JJ, Bellot J, Claramonte P, Ismail MM, Alió JL. Laser in situ keratomileusis to correct high myopia. J Cataract Refract Surg. 1997;23(3):372-85.

10. Dulaney DD, Barnet RW, Perkins SA, Kezirian GM. Laser in situ keratomileusis for myopia and astigmatism: 6 month results. J Cataract Refract Surg. 1998;24(6):758-64.

11. Charman WN, Chateau N. The prospects for super-acuity: limits to visual performance after correction of monochromatic ocular aberration. Ophthalmic Physiol Opt. 2003;23(6):479-93.

12. Williams D, Yoon GY, Porter J, Guirao A, Hofer H, Cox I. Visual benefit of correcting higher order aberrations of the eye. J Refract Surg. 2000;16(5):S554-9.

13. Boxer Wachler BS, Durrie DS, Assil KK, Krueger RR. Role of clearance and treatment zones in contrast sensitivity: significance in refractive surgery. J Cataract Refract Surg. 1999;25(1):16-23.

14. McLeod SD. Beyond Snellen acuity: the assessment of visual function after refractive surgery. Arch Ophthalmol. 2001;119(9):1371-3.

15. Pesudovs K, Marsack JD, Donnelly WJ 3rd, Thibos LN, Applegate RA. Measuring visual acuity-mesopic or photopic conditions, and high or low contrast letters? J Refract Surg. 2004;20(5):S508-14.

16. Lawless MA, Hodge C, Rogers CM, Sutton GL. Laser in situ keratomileusis with Alcon CustomCornea. J Refract Surg. 2003;19(6):S691-6.

17. Mrochen M, Kaemmerer M, Seiler T. Clinical results of wavefront-guided laser in situ keratomileusis 3 months after surgery. J Cataract Refract Surg. 2001; 27(2):201-7.

18. Dausch D, Dausch S, Schröder E. Wavefront-supported photorefractive keratectomy: 12-month follow-up. J Refract Surg. 2003;19(4):405-11.

19. Montés-Micó R, Muñoz G, Albarrán-Diego C, Rodríguez-Galietero A, Alió JL. Corneal aberrations after astigmatic keratotomy combined with laser in situ keratomileusis. J Cataract Refract Surg. 2004;30(7):1418-24.

20. Pallikaris IG. Quality of vision in refractive surgery. Barraquer Lecture 1997. J Refract Surg. 1998;14(5):549-8.

21. Quesnel NM, Lovasik JV, Ferremi C, Boileau M, Ieraci C. Laser in situ keratomileusis for myopia and the contrast sensitivity function. J Cataract Refract Surg. 2004;30(6):1209-18. Comment in: J Cataract Refract Surg. 2005; 31(6): 1087-8.

22. Lackner B, Pieh S, Schmidinger G, Hanselmayer G, Simader C, Reitner A, Skorpik C. Glare and halo phenomena after laser in situ keratomileusis. J Cataract Refract Surg. 2003;29(3):444-50.

23. Carr JD, Stulting RD, Sano Y, Thompson KP, Wiley W, Waring GO $3^{\text {rd }}$. Prospective comparison of single-zone and multizone laser in situ keratomileusis for the correction of low myopia. Ophthalmology. 1998;105(8):1504-11.

24. Pettit GH, Campin JA, MacDonald MB, Krueger RR. Customized ablation using the Alcon CustomCornea platform. In: Krueger RR, Applegate RA, MacRae SM, editors. Wavefront customized visual correction: the quest for super vision II. Thorofare; 2004. p.217-25.

25. Liang J, Koch DD. Customized ablation using the VISX WaveScan system and the VISX S4 ActiveTrak excimer laser. In: Krueger RR, Applegate RA, MacRae SM, editors. Wavefront customized visual correction: the quest for super vision II. Thorofare: Slack Incorporated; 2004. p.227-33.

26. MacRae SM, Slade S, Durrie DS, Cox I. Customized ablation using the Bausch \& Lomb Zyoptix System. In: Krueger RR, Applegate RA, MacRae SM, editors. Wavefront customized visual correction: the quest for super vision II. Thorofare: Slack Incorporated; 2004. p.235-41.

27. Slade S. Contralateral comparison of Alcon CustomCornea and VISX CustomVue wavefront-guided laser in situ keratomileusis: one-month results. J Refract Surg. 2004;20(5):S601-5.

28. Awwad ST, El-Kateb M, Bowman RW, Cavanagh HD, McCulley JP. Wavefront-guided laser in situ keratomileusis with the Alcon CustomCornea and the VISX CustomVue: three-month results. J Refract Surg. 2004;20(5):S606-13.

29. Kaiserman I, Hazarbassanov R, Varssano D, Grinbaum A. Contrast sensitivity after wave front-guided LASIK. Ophthalmology. 2004;111(3):454-7.

30. Rani A, Balasubramanya R, Sharma N, Tandon R, Vajpayee RB, Dada VK, Singh R. Outcomes after laser in situ keratomileusis retreatment in high myopes. J Refract Surg. 2003;19(2):159-64. 